\title{
Cold uniform spherical collapse revisited
}

\author{
M. Joyce*, B. Marcos ${ }^{\dagger}$ and F. Sylos Labini**,‡ \\ ${ }^{*}$ Laboratoire de Physique Nucléaire et de Hautes Energies, UMR 7585, Université Pierre et Marie Curie - \\ Paris 6, 75252 Paris Cedex 05, France \\ ${ }^{\dagger}$ Laboratoire J.-A. Dieudonné, UMR 6621, Université de Nice - Sophia Antipolis, Parc Valrose 06108 Nice \\ Cedex 02, France \\ ** “E. Fermi" Center, Via Panisperna 89 A, Compendio del Viminale, I-00184 Rome, Italy \\ $¥$ ISC-CNR, Via dei Taurini 19, I-00185 Rome, Italy
}

\begin{abstract}
We report results of a study of the Newtonian dynamics of $N$ self-gravitating particles which start in a quasiuniform spherical configuration, without initial velocities. These initial conditions would lead to a density singularity at the origin at a finite time when $N \rightarrow \infty$, but this singularity is regulated at any finite $N$ (by the associated density fluctuations). While previous studies have focussed on the behaviour as a function of $N$ of the minimal size reached during the contracting phase, we examine in particular the size and energy of the virialized halo which results. We find the unexpected result that the structure decreases in size as $N$ increases, scaling in proportion to $N^{-1 / 3}$, a behaviour which is associated with an ejection of kinetic energy during violent relaxation which grows in propertion to $N^{1 / 3}$. This latter scaling may be qualitatively understood, and if it represents the asymptotic behaviour in $N$ implies that this ejected energy is unbounded above. We discuss also tests we have performed which indicate that this ejection is a mean-field phenomenon (i.e. a result of collisionless dynamics).
\end{abstract}

Keywords: N-body simulations; virialization; spherical collapse

PACS: 95.30.-k, 95.30.Sf, 05.20.-y

\section{INTRODUCTION}

We will discuss here numerical simulations, and some simple analytical results, of what appears at first sight perhaps a very simple initial condition which one might consider naturally in the study of the virialization of self-gravitating systems: a large number $N$ of point particles are randomly distributed in a sphere, and evolved from a cold (i.e. zero velocity) start. The "simplicity" of the family of initial conditions is that they are characterized by a single parameter, the particle number $N$ (as the system is open the units may be defined by the system size, its total mass and Newton's constant $G$ ). Further as $N \rightarrow \infty$ the initial conditions tends to that considered as a starting point for analyses of nonlinear structure formation in cosmology — the "cold spherical collapse" model, in which a perfectly uniform spherical overdensity embedded in an expanding universe is considered. A random sampling with particles of such a flat density profile is the simplest discrete realisation of this theoretical model one can envisage, and might thus be expected perhaps to be the subject of much study. In practice this initial condition has been but little studied - references to the few previous studies will be given below - because of the intrinsic difficulty in its numerical integration which is related to a property of the uniform limit: as $N \rightarrow \infty$ the evolution of the system leads to a singularity after a finite time, as all the mass arrives at the origin after a time $t_{\text {coll }} \sim 1 / \sqrt{G \rho_{0}}$ where $\rho_{O}$ is the initial mass density. While for any finite $N$ system the singularity does not occur, the typical size of the region the system contracts to before "turn-around" decreases as $N$ increases, and the typical particle velocities grow. This makes the numerical integration very costly, and limits greatly the accessible particle $N$ compared to other warm or less homogeneous initial conditions.

One motivation for this study comes thus from the "uniform spherical collapse model": when calculating predictions for the masses and abundances of halos in the framework, one of the critical assumptions is that all the mass and energy in the initially collapsing region is ultimately virialized in the collapsed structure. The question arises as to whether this is generically true independently of the initial conditions. In the present case - which we have noted is, in a simple sense, the "closest" initial condition to the exactly uniform case - it turns out, interestingly, that this is not a good approximation: as we will describe, the violence of the collapse leads to an ejection of energy from the system (as kinetic energy of particles which escape with positive energy). Our numerical analysis, coupled to an analytical scaling argument, lead us to conclude that the ejected energy is in fact unbounded above as $N$ increases, so that these collapses can be characterized as causing "explosions", with purely Newtonian gravitational physics.

The initial motivation for our study of these initial conditions was, however, another one: this class of initial 
conditions poses issues about discreteness (i.e. N-dependent, non-collisional) effects in an interesting way. The aim of $\mathrm{N}$-body simulations in cosmology (and indeed, most such simulations in astrophysics more generally) is to represent the collisionless limit, which means that the results of such simulations should be explicitly independent of $N$ (or depend very weakly on them at the $N$ considered). In practice this means that, if the dynamics is indeed collisionless, it should be possible to define an appropriate extrapolation of $N$ which gives stable macroscopic results.In the present class of initial conditions it is clear that the simple extrapolation of $N \rightarrow \infty$ described above does not give $N$ independent results - as we have already indicated we find explicit $N$ dependences of, for example, the final size and binding energy of the virialized structure. There are then two possibilities: either the physics of this ejection is not in fact collisionless - which one would perhaps imagine might be the case as the particles do coherently contract into a very small region - or else the extrapolation in $N$ must be performed in a different way. Through a careful study which we will summarize below we have shown that it the latter which is the correct explanation. The physical reason is that the $N$ dependence which appears in the final state when one extrapolates "naively" is in fact a result of the fact that $N$ controls the amplitude of the fluctuations about uniformity, which are the physically relevant quantities. The appropriate extrapolation to the collisionless limit is that in which the particle number is increased, while keeping these fluctuations fixed. This extrapolation requires the introduction of an additional characteristic scale, which is the scale above which the fluctuations are kept (approximately) invariant. The existence of the collisionless limit is, conversely, a result of the fact that the dynamics is only sensitive to fluctuations above some finite scale. The study of this problem for these initial conditions thus allow one to understand some of the subtleties which may be involved in practically testing for collisionality effects in $N$ body systems. This is in principle an important issue in cosmological simulations (see [1] for a detailed discussion).

This proceedings essentially summarizes synthetically results reported in much greater detail in [2]. Because of space limitations we refer the reader to this paper for a fuller set of references on previous studies of virialization, giving here only the essential references, in particular to the few previous detailed studies of these specific initial conditions $[3,4,5,6]$.

\section{COLD COLLAPSE THEORY}

We recall first the limit $N \rightarrow \infty$. The radial position $r(t)$ of a test particle in an (idealized) exactly uniform spherical distribution of purely self-gravitating matter of initial density $\rho_{0}$ and initially at rest (at time $t=0$ ) is simply given by the homologous rescaling

$$
r(t)=R(t) r(0)
$$

where the scale factor $R(t)$ may be written in the standard parametric form

$$
\begin{aligned}
& R(\xi)=\frac{1}{2}(1+\cos (\xi)) \\
& t(\xi)=\frac{\tau_{s c m}}{\pi}(\xi+\sin (\xi)),
\end{aligned}
$$

with $\tau_{s c m} \equiv \sqrt{\frac{3 \pi}{32 G \rho_{0}}}$. At the time $\tau_{s c m}$ the system collapses to a singularity, and physical quantities diverging. More specifically, taking $\xi=\pi-\varepsilon$ and expanding to leading order in $\varepsilon$ gives $\left(t-\tau_{s c m}\right) \sim \varepsilon^{3}$, we have that $R(t) \sim\left[t-\tau_{s c m}\right]^{2 / 3}$ and therefore the test particle velocities $v(t)$, proportional also to the initial radius $r(0)$, scale as $v(t) \sim\left[t-\tau_{s c m}\right]^{-1 / 3}$

$N$ randomly placed particles in a spherical volume can be treated, up to some time and at sufficiently large scales, as a perturbed version of this uniform limit. Consider the approximation in which we treat the perturbations as if they evolve also in an infinite contracting system (i.e. neglect the effect of the boundaries on the evolution of the density perturbations). In the manner standard in cosmology (for the case of an expanding universe) one can then consider the fluid limit for the system and solve the appropriate equations perturbatively (see e.g. [7]). In the eulerian formalism this gives, at linear order, a simple equation for $\delta(\mathbf{x})$, the density fluctuation (with respect to the mean density):

$$
\ddot{\delta}+2 H \dot{\delta}-4 \pi G \rho_{0} \delta=0
$$

where $H(t)=\dot{R} / R$ (dots denotes derivatives with respect to time) is the contraction ("Hubble") rate. These equations are derived in "comoving" coordinates $\mathbf{x}=\mathbf{r} / R(t)$, where $\mathbf{r}$ are the physical vector positions. Note that $R \dot{\mathbf{x}} \equiv=\dot{\mathbf{r}}-\dot{R} \mathbf{x}$ i.e., $R(t) \dot{\mathbf{x}}(t)$ is the "peculiar" velocity with respect to the "Hubble flow". 
It is straightforward to show, from Eq. (3), that, in the limit $R \ll 1$,

$$
\delta(R) \sim R^{-3 / 2} .
$$

This is simply the usual decaying mode of the expanding EdS universe, which becomes the dominating growing mode in the contracting case.

The singular behaviour of the spherical collapse is regulated by the fluctuations present at any finite $N$ in the initial conditions we study. A simple estimate of the scale factor $R_{\min }$ at which one expects the spherical collapse model to break down completely may be obtained by assuming that this will occur when fluctuations at some scale (e.g. of order the size of the system) go non-linear. For Poisson distributed particles we have a mass variance proportional to $N$, and so the amplitude of the initial normalized density fluctuations is proportional to $1 / \sqrt{N}$. Using the growth law given in Eq. (4), we can infer [3, 4].

$$
R_{\min } \propto N^{-1 / 3} .
$$

\section{NUMERICAL RESULTS}

Details of our numerical simulations, performed using the publicly available and widely used GADGET2 code [8,9], are given in [2]. Our study covers a range of $N$ between several hundred and several hundred thousand. We mention here just one important consideration: instead of the exact Newtonian potential, the code employs, for numerical reasons, a two-body potential which is exactly Newtonian above a finite "smoothing length" $\varepsilon$, and regularized below this scale to give a force which is 1) attractive everywhere and 2) vanishes at zero separation. The (complicated) analytic expression for the smoothing function may be found in [9]. With this modified force the code does not integrate accurately trajectories in which particles have close encounters, which lead to very large accelerations and thus the necessity for very small time steps (which is numerically costly). However, on the (short) time scales we consider such trajectories should not play any significant role in modifying the macroscopic properties we are interested in. The results shown below correspond to a constant value of $\varepsilon$ in all simulations, a few times smaller than $\ell$ in the largest $N$ simulation. As we will detail further below when we discuss the collisionless limit for our system, we have tested our results in particular for stability when $\varepsilon$ is extrapolated to smaller values, and we interpret them to be indicative, on the relevant time scales, of the $\varepsilon=0$, i.e., the exact Newtonian limit ${ }^{1}$.

Qualitatively the evolution we observe in all our simulations is the same, and like that well known in both astrophysics, and, more generally, in statistical physics for systems with long-range attractive interactions from subvirial initial conditions of this type (i.e. with an initial virial ratio larger than -1) : the system first contracts and relaxes "violently" (i.e. on timescales of order the dynamical time scale $\tau_{s c m}$ ) to give a virialized, macroscopically stationary, state (see e.g. [10]).

\section{Minimal size: phenomenology}

The existing studies in the astrophysical literature of this class of initial conditions [3,4] focus on how the singular collapse of the uniform spherical collapse model is regulated at finite $N$, and in particular on the scaling with $N$ in numerical simulations of the minimal size reached by the system. Indeed in the study of [3] the "points" represent masses with extension (e.g. proto-stars) and the central question the authors wish to address is whether these masses survive or not the collapse of a cloud of which they are the constituents. This minimal radius $R_{\min }$ may be defined in different ways, e.g., as the minimal value reached by the radius, measured from the center of mass, enclosing $90 \%$ of the mass. Alternatively it can be estimated as the radius inferred from the potential energy of the particles, the minimal radius corresponding to the maximal negative potential energy. The behavior of $R_{\min }$, determined by the first method, as a function of $N$ is shown in Fig. 1 . The fitted line $R_{\min } \propto N^{-1 / 3}$ is the theoretical behavior predicted by the simple arguments given in the previous section. Agreement with this simple prediction has also been verified in both [3] and [4], the latter for an $N$ as large as $10^{7}$.

\footnotetext{
${ }^{1}$ As a test we have also performed simulations using a code with a direct $N^{2}$ summation, and without any smoothing. For the range of $N$ (up to a few thousand) for which we can run this code over the same physical time-scale, we find excellent agreement with the results obtained with GADGET2 with the smoothing we have adopted (see [2] for exact parameter values, as well as details of energy conservation etc.).
} 


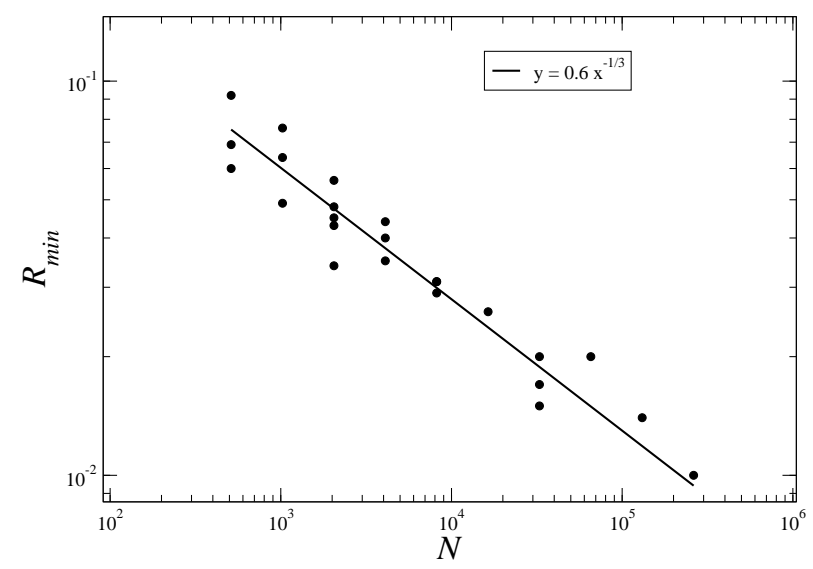

FIGURE 1. Behaviour of the minimal radius $R_{\min }$ attained, determined as described in text, as a function of $N$. The solid line is the best fit to the prediction of Eq. (5).

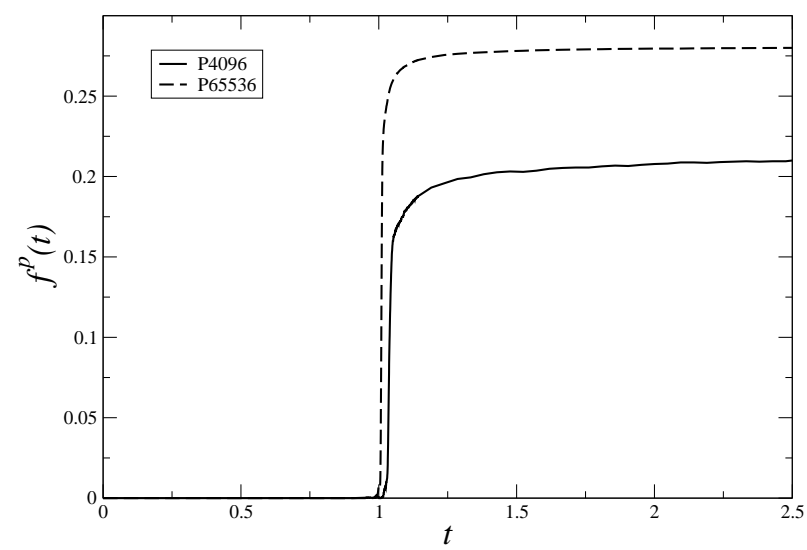

FIGURE 2. The behavior of $f^{p}(t)$, the fraction of the particles with positive energy, as a function of time for two different simulations.

\section{Mass ejection}

All particles start with a negative energy, but a finite fraction can in principle end up with a positive energy and escape from the system completely. While evidently the ejected mass is bounded above by the initial mass, the ejected energy is, in principle, unbounded above as the gravitational self energy of the bounded final mass is unbounded below.

Shown in Fig. 2 is the fraction $f^{p}$ of the particles with positive energy as a function of time in two different simulations, while Fig. 3 shows the asymptotic value of $f^{p}$ in each simulation as a function of $N$ (i.e. the value attained on the "plateau" in each simulation after a few dynamical times, corresponding to particles which are definitively ejected on these time scales). While some previous works (see [2] for references) have noted the ejection of some small fraction of the mass in similar cases, the significance of the energy ejection as $N$ increases, and its $N$ dependence, has not previously been documented. Theoretical studies of the ejection of mass from a pulsating spherical system which is qualitatively similar to that described below for the ejection observed here - can be found in [11, 12].

Although $f^{p}$ fluctuates in different realizations with a given particle number, it shows a very slow, but systematic, increase as a function of $N$, varying from approximately $15 \%$ to almost $35 \%$ over the range of $N$ simulated. A reasonably good fit is given by

$$
f^{p}(N) \approx a+b \log (N),
$$

where $a=0.048$ and $b=0.022$. Alternatively it can be fit quite well (in the same range) by a power law $f^{p} \approx 0.1 N^{0.1}$. Note that these fits cannot, evidently, be extrapolated to arbitrarily large $N$ (as the mass ejected is bounded above), and thus our study does not actually definitively determine the asymptotic large $N$ behavior of this quantity despite the 


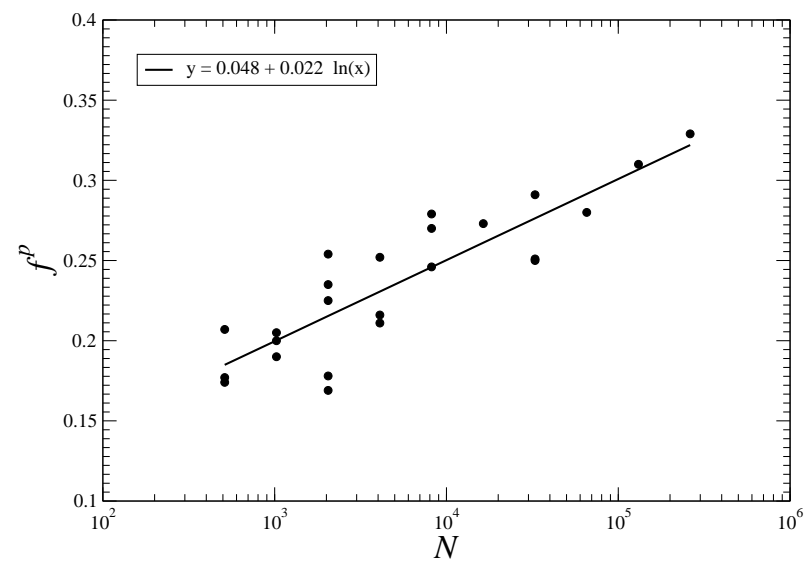

FIGURE 3. Behavior of the fraction of ejected particles as a function of the number $N$ of simulation particles in the system. The solid line is the phenomenological fit given by Eq. (6).

large particle numbers simulated. As we will discuss briefly below, however, the mechanism we observe for this mass ejection leads us to expect that the value of $f^{p}$ should saturate when $f^{p} \sim 0.5$.

\section{Energy ejection}

Let us now consider the energy carried away by these particles. Using total energy conservation, and the fact that both the final potential energy of the ejected particles and that associated with the interaction of the bound and ejected particles is negligible, we have

$$
E_{0}=W^{n}+K^{p}+K^{n}
$$

where $E_{0}$ is the initial energy of the system, $W^{n}$ and $K^{n}$ are the potential and kinetic energy of the particles which are finally bound, and $K^{p}$ is the kinetic energy of the escaping particles. Further, since the bound particles in the QSS are virialized we have

$$
2 K^{n}+W^{n}=0 .
$$

Thus we have

$$
W^{n}=-2 K^{n}=2\left(E_{0}-K^{p}\right)
$$

Fig. 5 shows the ratio $K^{p} / f^{p}$, i.e., the kinetic energy per unit ejected mass, as a function of $N$. Its behavior is fit very well by $K^{p} / f^{p} \propto N^{1 / 3}$. Note that for the largest values of $N$ simulated $K^{p}$ is almost ten times the initial (potential) energy $E_{0}$ of the system. It follows that we have the approximate behavior $W^{n} \propto-N^{-1 / 3}$ (when we neglect the slow observed variation with $N$ of $f^{p}$ ).

\section{Properties of virialized "halo"}

The dependence of the ejected energy on $N$ implies that the macroscopic properties of the virialized structure (which we refer to as a "halo" in the sense current in cosmology) also depend on $N$. Studying the radial density profiles of the (approximately spherically symmetric) halos we find that they can always be fit well by the simple functional form

$$
n(r)=\frac{n_{0}}{\left(1+\left(\frac{r}{r_{0}}\right)^{4}\right)}
$$

This form of the profile agrees well with that found in previous studies for collapses from low initial virial ratio (see [2] for references). The $N$ dependence we find here is encoded in that of the two parameters $n_{0}$ and $r_{0}$, which we find 


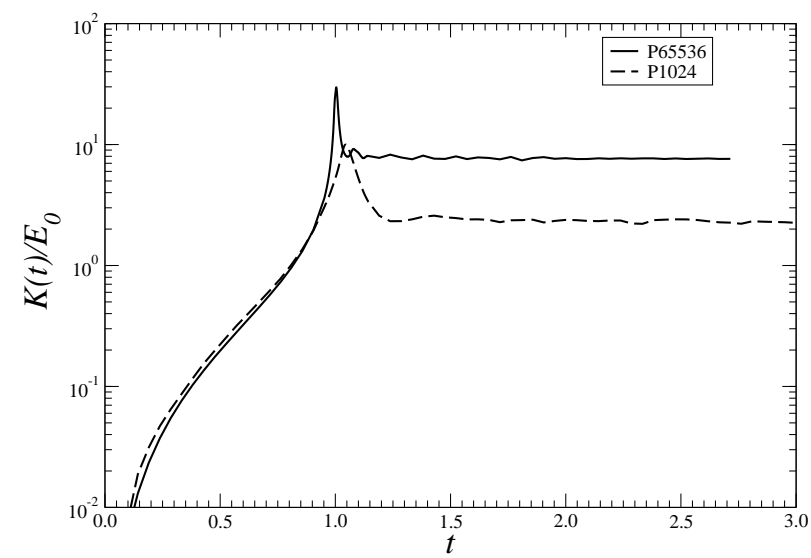

FIGURE 4. Behaviour of the total kinetic energy for two simulations with different number of particles.

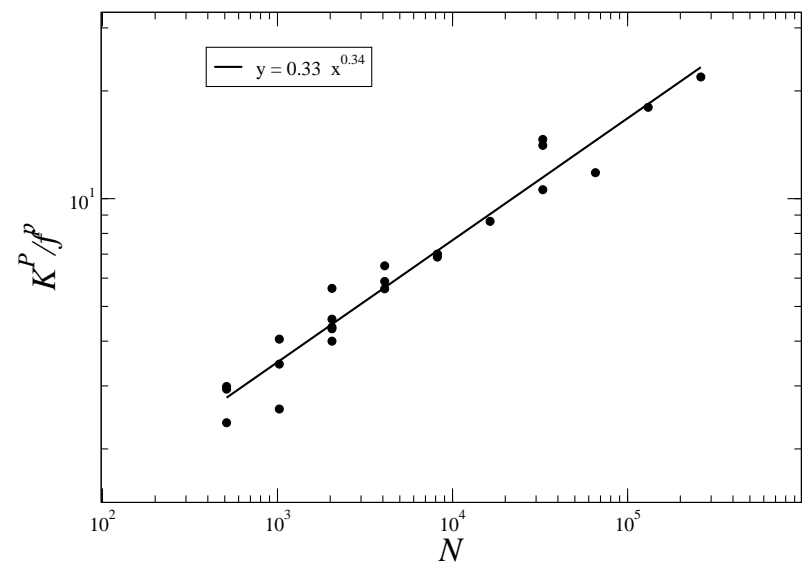

FIGURE 5. Observed behaviour of the ratio $K^{p} / f^{p}$ as a function of particle number $N$.

are well fit by $r_{0} \propto N^{-1 / 3}$ and $n_{0} \propto N^{2}$. In Fig. 6 we show the density profiles for various simulations with different $N$ where the axes have been rescaled using these behaviors.

It is simple to show that these scalings with $N$ of $n_{0}$ and $r_{0}$ are simply those which follow from those just given for $f^{p}$ and $W^{n}$ : using the ansatz Eq. (10) one has that the number $N^{n}$ of bound particles is proportional to $n_{0} r_{0}^{3}$ while the potential energy $W^{n}$ is proportional to $m^{2} n_{0}^{2} r_{0}^{5}$ where $m$ is the mass of a particle. The best fit behaviors for $r_{0}$ and $n_{0}$ thus correspond, since $m \propto 1 / N$, to $N^{n} \sim N$ (i.e. a constant bound mass, and therefore a constant ejected fraction of the mass $f^{p}$ ) and $W^{n} \sim N^{1 / 3}$. More detailed fits to $n_{0}$ and $r_{0}$ show consistency also with the very slow variation of $f^{p}$ observed. In summary the $N$ dependence of the virialized structure manifests itself to a very good approximation simply in a scaling of its characteristic size in proportion to $R_{\min }$, the minimal radius attained in the collapse (which, as we have seen, is proportional to the initial inter-particle separation).

\section{FURTHER ANALYSIS AND DISCUSSION}

\section{Mechanism of energy ejection}

We limit ourselves here to a very brief qualitative description of a detailed study we have performed (see [2]) of the evolution of the system during collapse and the mechanism which leads to the mass and energy ejection. It is simple to establish that the probability of ejection is closely correlated with particles' initial radial positions, with essentially particles initially in the outer shells being ejected. The reason why this is so can be understood as follows. Firstly, 


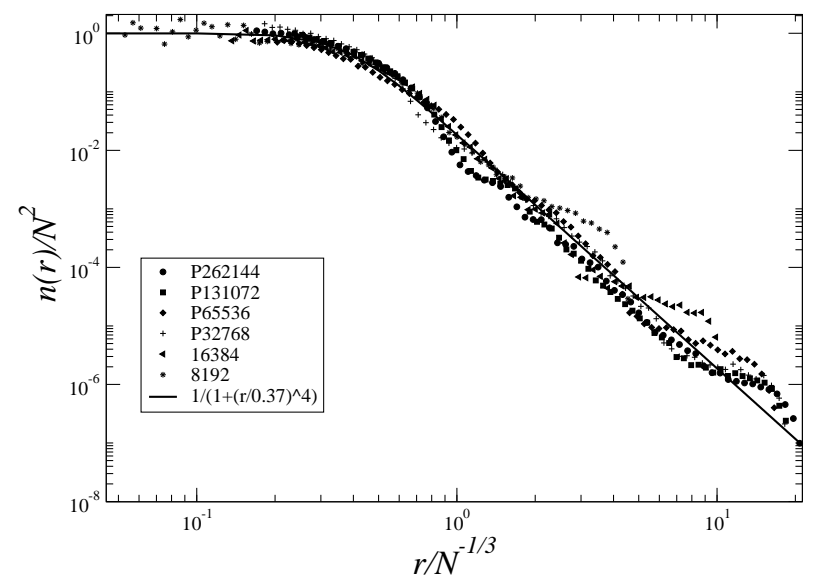

FIGURE 6. Density profile of the virialized structure at a time $t \approx 4 \tau_{s c m}$ for simulations with different number of particles. The y-axis has been normalized by $N^{2}$ and the x-axis by $N^{-1 / 3}$ (see text for explanations). The behaviour of Eq. (10) is shown for comparison.

particles closer to the outer boundary systematically lag (in space and time) with respect to their uniform spherical collapse trajectories more than those closer to the center. This is an effect which arises from the fact that, when mass moves around due to fluctuations about uniformity, there is in a radial shell at the boundary no average inward flux of mass to compensate the average outward flux. The mean mass density thus seen by a particle in such a shell decreases, leading to a slowdown of its fall towards the origin. This "lag" with respect to particles in the inner shells propagates in from the boundaries with time, leading to a coherent relative lag of a significant fraction of the mass by the time of maximal compression. Secondly, these lagging particles are then ejected as they pick up energy, in a very short time around the collapse, as they pass through the time-dependent potential of the particles initially closer to the center, which have already collapsed and "turned around". This is illustrated in Fig. 7, which shows, for a simulation with $N=131072$ particles, the temporal evolution of the components of the mass which are asymptotically ejected or bound. More specifically the plot shows the evolution of $v_{e}$ (and $v_{b}$ ) which is the average of the radial component of the velocity for the ejected (and bound) particles, and also $\mathrm{e}_{e}$ (and $\mathrm{e}_{b}$ ) which is the mean energy per ejected (and bound) particle (i.e. the average of the individual particle energies). The behaviors of $v_{e}$ and $v_{b}$ show clearly that the ejected particles are those which arrive on average late at the center of mass, with $v_{e}$ reaching its minimum after the bound particles have started moving outward. Considering the energies we see that it is in this short time, in which the former particles pass through the latter, that they pick up the additional energy which leads to their ejection. Indeed the increase of $e_{e}$ sets in just after the change in sign of $v_{b}$, i.e., when the bound component has (on average) just "turned around" and started moving outward again. The mechanism of the gain of energy leading to ejection is simply that the outer particles, arriving later on average, move through the time dependent decreasing mean field potential produced by the re-expanding inner mass. Assuming that the fraction of the lagging mass is independent of $N$, an analysis of the scaling (see [2]) of the relevant characteristic velocity/time/length scales allows one to infer the observed scaling of the ejected energy with $N$. Quantitatively we have not been able to explain, on the other hand, the observed $N$ dependence of the lagging mass, which should determine the $N$ dependence of $f^{p}$. Given, however, that it is determined by a lag of the outer mass relative to that of the inner mass, it seems clear that, as required, the mechanism observed will naturally lead it to saturate at a fixed fraction, of order one, as $N$ increases arbitrarily.

\section{Is dynamics collisionless?}

The mechanism we have described for the mass and energy ejection is clearly of the "mean-field" type — the particles have been considered to move in the field produced by the bulk of the mass and no role has made ascribed, notably, to collisions with individual particles. As the system has contracted so much before re-expanding, particular care should evidently be taken in verifying that this is indeed the case, i.e., that the simulations do indeed represent well the collisionless limit. As discussed briefly this is none other than a particular case of a question which can be posed about gravitational $N$-body simulation, but one which shows the subtleties there may be in defining an appropriate 


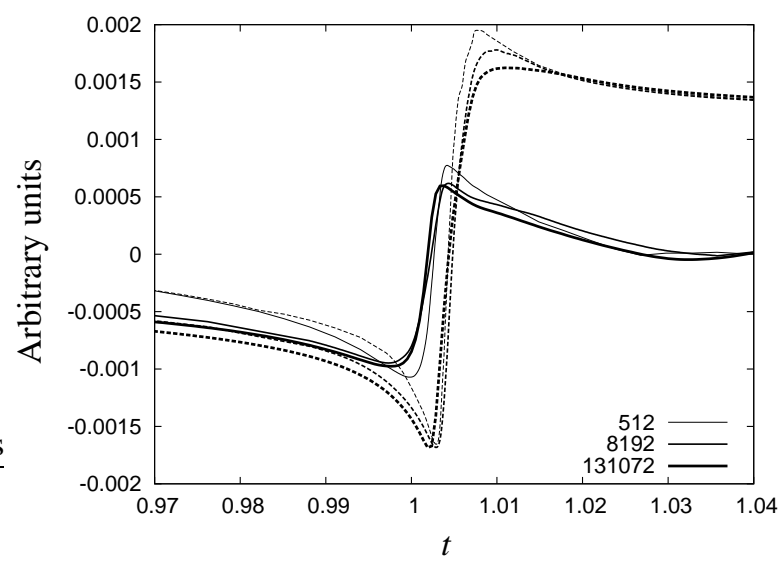

FIGURE 7. Radial velocity, and average energy per particle, as a function of time, of particles which are bound/ejected at the end of the simulation of 131072 particles. The energy of the particles has been arbitrarily rescaled.

numerical extrapolation to provide a clear answer. More precisely the question is whether the system approximates well the evolution which would be obtained from a set of "collisionless Boltzmann equation" coupled to the Poisson equation, i.e. the Vlasov-Poisson (VP) equations. Is this the case? To determine whether it is we need to understand how we can test for its validity. As the VP limit is an appropriate $N \rightarrow \infty$ limit for the system, this means specifying precisely how this limit should be taken. We can then extrapolate our numerical simulations to larger $N$ to test for the stability of results.

Firstly it is clear that the appropriate extrapolation for the system we have studied is not the naive limit $N \rightarrow \infty$, i.e., in which we simply increase the number of Poisson distributed particles: we have explicitly identified macroscopic $N$ dependencies in fundamental quantities, so the results at any given $N$ do not approximate those at any other $N$, and indeed do not converge towards any $N$-independent behavior.

Formal proofs of the validity of the VP limit [13] for a self-gravitating system require, however, that the singularity in the gravitational force at zero separation be regulated when the limit $N \rightarrow \infty$ is taken. This suggests we should take the limit $N \rightarrow \infty$ while keeping fixed a smoothing scale, like the $\varepsilon$ we have introduced in our simulations. Doing so we would indeed expect to obtain a well defined $N$-independent result, corresponding to the uniform spherical model with such a regularization of the force: the sphere will not collapse below a radius of order $\varepsilon$, as the force is then weaker than the Newtonian force (and goes asymptotically to zero). One would then expect to obtain, for sufficiently large $N$, a final state which is well defined and $N$ independent, but dependent on the scale of the regularization and indeed even on the details of its implementation.

This limit is not the VP limit relevant here. We have indeed introduced a regularization of the force, but this has been done, as we have discussed, only for reasons of numerical convenience, and our criterion for our choice of $\varepsilon$ is that it be sufficiently small so that our numerical results are independent of it. Our results are thus, a priori, independent of the scale $\varepsilon$ (and of how the associated regularization is implemented). To illustrate this we show in Fig. 8 the evolution of $f^{p}$ (the fraction of particles with positive energy) as a function of time, in simulations from identical initial conditions with $N=32768$ particles in which only the value of $\varepsilon$ has been varied, through the values indicated. Other quantities we have considered show equally good convergence as $\varepsilon$ decreases. Note that for the given simulation the mean interparticle distance in our units is $\ell=0.016$, so that the convergence of results is attained once $\varepsilon$ is significantly less than $\ell$. As we have seen, the minimal size reached by the collapsing system scales in proportion to $\ell$. We interpret the observed convergence as due to the fact that the evolution of the system is determined primarily by fluctuations on length scales between this scale and the size of the system. Once $\varepsilon$ is sufficiently small to resolve these length scales at all times, convergence is obtained.

Given the essential role played by fluctuations to the mean density in determining the final state, it is clear that only an extrapolation of $N$ which keeps the fluctuations in the initial conditions fixed can be expected to leave the macroscopic results invariant. Any change in $N$ necessarily leads, however, to some change in the fluctuations. If, however, as indicated by the above results, there is a minimal length scale in the initial conditions for "relevant" fluctuations, we expect an extrapolation of $N$ which leaves fluctuations above this minimal scale unchanged to give stable results. 


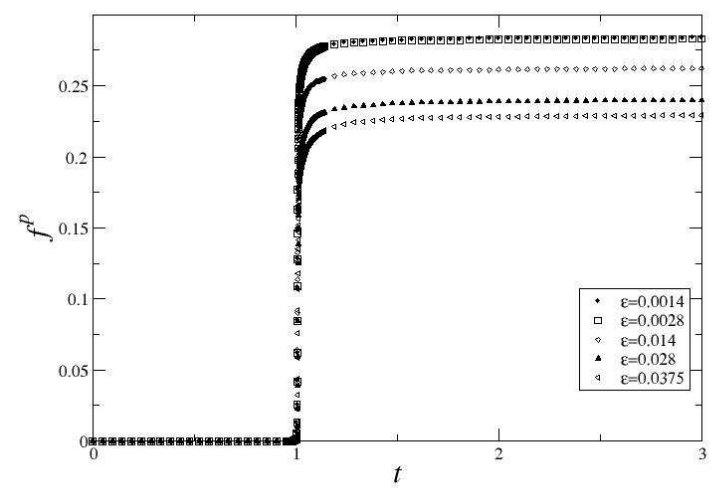

FIGURE 8. Evolution of the fraction of the mass with positive energy for simulations with $N=32768$ for the different values indicated of the smoothing parameter $\varepsilon$.

Such an extrapolation for our initial conditions can be defined as follows. Starting from a given Poissonian initial condition of $N$ particles in a sphere, we create a configuration with $N^{\prime}=n N$ particles by splitting each particle into $n$ particles in a cube of side $2 r_{s}$, centered on the original particle. The latter particles are distributed randomly in the cube, with the additional constraint that their center of mass is located at the center of the cube, i.e., the position of the center of mass is conserved by the "splitting". In this new point distribution, which has the same mean mass density as the original distribution, fluctuations on scales larger than $r_{s}$ are essentially unchanged compared to those in the original distribution, while fluctuations around and below this scale are modified (see [14] for a detailed study of how fluctuations are modified by such "cloud processes".). We have performed this experiment for a Poisson initial condition with $N=4096$ particles, splitting each particle into eight $(n=8)$ to obtain an initial condition with $N^{\prime}=32768$ particles. Results are shown in Fig. 9 for the ejected mass as a function of time, for a range of values of the parameter $r_{s}$, expressed in terms of $\ell$, the mean inter-particle separation (in the original distribution). While for $r_{s}=0.8 \ell$ the curve of ejected particles is actually indistinguishable in the figure from the one for the original distribution, differences can be seen for the other values, greater discrepancy becoming evident as $r_{s}$ increases. This behavior is clearly consistent with the conjecture that the macroscopic evolution of the system depends only on initial fluctuations above some scale, and that this scale is of order the initial inter-particle separation $\ell$. And, as anticipated, this translates into an $N$ independence of the results when $N$ is extrapolated in this way for an $r_{s}$ smaller than this scale.

This prescription for the VP limit can be justified theoretically using a derivation of this limit through a coarsegraining of the exact one particle distribution function over a window in phase space (see e.g. [15]). The VP equations are obtained for the coarse-grained phase space density when terms describing perturbations in velocity and force below the scale of the coarse-graining are neglected. A system is thus well described by this continuum VP limit if the effects of fluctuations below some sufficiently small scale play no role in the evolution. The definition of the limit thus requires explicitly the existence of such a length scale, and the limit is approached in practice when the mean inter-particle distance becomes much smaller than this scale. With the kind of procedure given we have defined not only an extrapolation of $N$ which gives stable results, but also a method of identifying this scale.

\section{Some conclusions}

The most surprising result of our study is that a simple initial configuration of particles interacting by Newtonian gravity only can liberate, in a time of order the dynamical time, an energy which is, apparently, unbounded above, growing approximately in proportion to $N^{1 / 3}$. We have given a physical description of this ejection which accounts for this scaling, and performed careful numerical tests to check that the evolution is indeed collisionless. This latter step required the definition of the appropriate numerical extrapolation, which in this case is quite non-trivial. This provides an illustration of the subtleties which may be involved in determining finite $N$ corrections to the collisionless limit, which we have argued elsewhere [1] are important notably in the context of cosmological simulations of dark matter. 


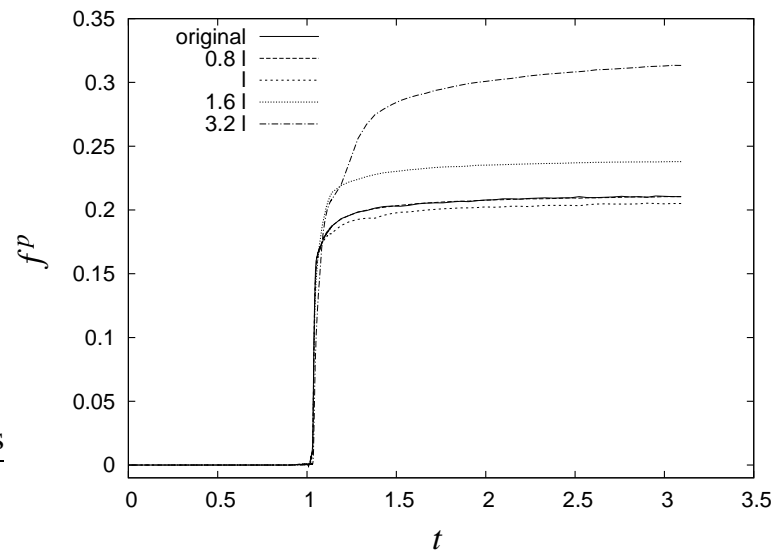

FIGURE 9. Evolution of the fraction of particles with positive energy as a function of time, for the different indicated values of the parameter $r_{s}$ described in text. The "original" initial conditions has 4096 particles while the others have 32768 particles. The curve for $r_{s}=0.8$ is not visible because it is superimposed on that of the original distribution.

In considering possible astrophysical relevance of these "classical gravitational explosions", a question which evidently arises is whether the energy ejection, which is associated essentially with the strong contraction during the collapse phase, is specific to initial conditions with spherical symmetry. In this respect we note that, although we expect smaller contraction factors for a generic initial condition, very large collapse factors may occur for non-symmetric initial conditions. This question has been considered at length in [4], which presents a detailed numerical study of the generalisation of the fluid SCM to axisymmetric and triaxial configurations. In the former singularities remain intact, and a relation $R_{\min } \propto N^{-1 / 6}$ is found empirically to replace the $R_{\min } \propto N^{-1 / 3}$ behaviour of the spherical case. In the triaxial case the collapse factors are found to be typically finite, but they can be very large and no upper bound is placed on them. In forthcoming work we will address the ejection of mass and energy also for initial conditions with non-zero initial velocities. The divergence we have identified will be regulated again in this case, but this does not exclude that significant energy ejection may occur and be relevant, in particular, to understanding the properties of the "remnant" virialized structure.

\section{ACKNOWLEDGMENTS}

We thank the Centro E. Fermi (Rome) for the use of computing resources. MJ thanks Steen Hansen for useful discussions, and the Istituto dei Sistemi Complessi, CNR, Rome, for hospitality during several visits.

\section{REFERENCES}

1. M. Joyce, B. Marcos, and T. Baertschiger, Mon. Not. R. Astron. Soc. 394, 751 (2009), arXiv : 0805.1457.

2. M. Joyce, B. Marcos, and F. Sylos Labini, Mon. Not. R. Astron. Soc 397, 775 (2009).

3. S. Aarseth, D. Lin, and J. Papaloizou, Astrophys. J. 324, 288-310 (1988).

4. C. Boily, E. Athanassoula, and P. Kroupa, Mon. Not. R. Astr. Soc. 332, 971-984 (2002).

5. O. Iguchi, Y. Sota, T. Tatekawa, A. Nakamichi, and M. Morikawa, Phys.Rev. E71, 016102 (2005).

6. O. Iguchi, Y. Sota, A. Nakamichi, and M. Morikawa, Phys.Rev. E73, 046112 (2006).

7. P. J. E. Peebles, The Large-Scale Structure of the Universe, Princeton University Press, 1980.

8. www.mpa-garching.mpg.de/gadget/right.html (2000).

9. V. Springel, N. Yoshida, and S. D. M. White, New Astronomy 6, 79-117 (2001), (also available on [8]).

10. J. Binney, and S. Tremaine, Galactic Dynamics, Princeton University Press, 1994.

11. M. David, and T. Theuns, Mon. Not. R. Astr. Soc. 240, 957-974 (1989).

12. T. Theuns, and M. David, Astrophys. Sp. Sci. 170, 276 (1990).

13. W. Braun, and K. Hepp, Comm. Math. Phys. 56, 101-113 (1977).

14. A. Gabrielli, and M. Joyce, Phys. Rev. E77, 031139 (2008).

15. T. Buchert, and A. Dominguez, Astron. Astrophys. 438, 443-460 (2005). 